Revue de droit comparé du travail et de la sécurité sociale

3 | 2019

Les migrations internationales de travail

\title{
La judiciarisation des conflits collectifs en Uruguay
}

\section{Hugo Barretto Ghione}

\section{(2) OpenEdition}

\section{Journals}

Édition électronique

URL : https://journals.openedition.org/rdctss/1513

DOI : $10.4000 /$ rdctss. 1513

ISSN : 2262-9815

Éditeur

Centre de droit comparé du travail et de la sécurité sociale

Édition imprimée

Date de publication : 1 novembre 2019

Pagination : 176-179

ISSN : 2117-4350

\section{Référence électronique}

Hugo Barretto Ghione, "La judiciarisation des conflits collectifs en Uruguay », Revue de droit comparé du travail et de la sécurité sociale [En ligne], 3 | 2019, mis en ligne le 01 novembre 2021, consulté le 13 novembre 2021. URL : http://journals.openedition.org/rdctss/1513 ; DOI : https://doi.org/10.4000/ rdctss. 1513 Licence Creative Commons Attribution - Pas d'Utilisation Commerciale - Pas de Modification 4.0 International. 


\title{
HUGO BARRETTO GHIONE
}

\author{
UNIVERSITÉ DE LA RÉPUBLIQUE
}

\section{LA JUDICIARISATION DES CONFLITS COLLECTIFS EN URUGUAY}

L'une des particularités des relations collectives de travail en Uruguay a longtemps consisté en l'absence de législation réglementant l'organisation syndicale et la grève. En revanche, la négociation collective a fait l'objet de diverses interventions du législateur, notamment avec l'établissement de salaires minimums par branche d'activité (conseils des salaires) mis en place par des organes tripartites selon une règle en vigueur depuis 1943.

Cette tradition, historiquement soutenue par un mouvement syndical réfractaire à toute intervention de l'État dans la vie syndicale et par les tenants d'une doctrine très influente (Américo Plá Rodríguez, Héctor-Hugo Barbagelata, Oscar Ermida Uriarte, Helios Sarthou, Osvaldo Mantero, etc.) a favorisé l'autonomie collective. Aussi, n'existe-t-il pratiquement pas d'autre norme dans ce domaine que celle de la Convention internationale du travail $n^{\circ} 87$ de l'OIT, ratifiée en 1953. En ce qui concerne la grève, il n'existe aucune définition légale ni procédure hétéronome permettant son exercice selon des formes typiques ou atypiques, l'occupation des lieux de travail constituant l'une des formes d'exercice de la grève les plus controversées.

À cet égard, l'article 4 du décret n 165/2006 reconnaît l'occupation du lieu de travail comme une forme de grève. Cet élargissement du concept a conduit les principales organisations d'employeurs à déposer plainte auprès du Comité de la liberté syndicale de l'OIT, alléguant que le droit de propriété et la liberté de travail ne sont pas respectés. Dans cette affaire, le Comité de la liberté syndicale a réaffirmé sa position traditionnelle en autorisant l'occupation des lieux de travail, à condition qu'elle se fasse de manière pacifique, que les non-grévistes et le propriétaire de l'entreprise puissent entrer dans l'établissement.

À l'instar de cette conception autonome des relations de travail, les conflits collectifs ne sont pas résolus par voie judiciaire, les tribunaux du travail n'ayant compétence que pour résoudre les conflits individuels du travail.

Néanmoins, malgré cette structure autonome, certains aspects des conflits collectifs du travail présentent des signes évidents de judiciarisation. Celle-ci semble répondre à deux processus quasi-simultanés qui définissent les lignes d'un interventionnisme évident dans le domaine des relations collectives de travail, à tel point qu'on peut y voir une modification des lignes traditionnelles de notre culture juridique. 
D'une part, on observe depuis quelques années la mise en place par le gouvernement de coalition de gauche, instauré en 2005, de mécanismes d'intervention législative visant à promouvoir la syndicalisation et la négociation collective. Cette intervention de l'État par la voie juridique présente des caractéristiques nettement différentes de celles que l'on retrouve dans la plupart des pays d'Amérique latine, dans la mesure où il s'agit d'une loi favorisant la liberté syndicale qui ne restreint ni la grève, ni les organisations syndicales ou encore la négociation collective.

Pour preuve de ce processus de soutien juridique à l'action syndicale, on peut citer: - les dispositions de la loi n 17940 qui reconnaît le droit au congé syndical, la perception des cotisations syndicales et les possibilités de communication du syndicat dans l'entreprise;

- la reconnaissance de l'occupation du lieu de travail comme modalité d'exercice du droit de grève;

- et la modification de la loi n 10449 sur les conseils de salaires, qui a autorisé la mobilisation de ces organes tripartites sur initiative des organisations de travailleurs et d'employeurs, et pas seulement sur décision de l'exécutif.

La deuxième forme d'interventionnisme se retrouve dans le processus de judiciarisation des relations collectives de travail, selon une tendance récente en Uruguay. On peut ainsi identifier au moins quatre types d'interventions du pouvoir judiciaire dans les conflits collectifs du travail, venant ainsi rompre avec la tradition non-interventionniste de l'État dans ce domaine.

1) La première rupture avec le modèle antérieur à 2005 (année du changement politique susmentionné) réside dans la possibilité de soumettre aux tribunaux du travail un conflit collectif résultant d'actes de discrimination antisyndicale commis par l'employeur contre des militants syndicaux. Ce mécanisme interdit la discrimination antisyndicale en établissant deux procédures judiciaires visant à assurer la réintégration du travailleur sur son lieu de travail (articles 1 et 2 de la loi $n^{\circ}$ 17940). Certains spécialistes, comme Helios Sarthou, se sont montré très critiques à l'égard de la judiciarisation du conflit qui implique de pouvoir recourir au pouvoir judiciaire pour faire valoir la liberté syndicale et obtenir la réintégration du travailleur.

2) Le débat concernant les droits des organisations syndicales minoritaires, notamment celui sur le droit à la négociation collective et sur le choix de l'organisation la plus représentative au niveau d'une entreprise, a marqué une deuxième rupture. II s'agit d'affaires portées par des syndicats minoritaires dans des entreprises publiques qui ont contesté la représentation des syndicats traditionnels. Deux arrêts de la Cour administrative, portant sur des affaires découlant de conflits dans des entreprises publiques, ont ainsi établi que les organisations syndicales minoritaires peuvent négocier collectivement avec l'entreprise mais ne peuvent pas signer une convention collective. Cette décision est contradictoire dans la mesure où elle reconnaît le droit de négocier, mais appelle le syndicat majoritaire à signer la convention collective (qui est le produit de la négociation), alors même qu'il n'a pas négocié la convention collective. Ce paradoxe évident ne peut s'expliquer que par une certaine inexpérience ou ignorance du juge administratif dans le domaine du travail. Cette méconnaissance des relations collectives de travail renforce l'opinion de la majorité des experts du droit du travail, qui considèrent que les juges ne devraient intervenir ni sur ces questions relatives à l'organisation syndicale, ni dans les processus de négociation. 
3) Une troisième tendance se dégage: la saisine du juge par les acteurs sociaux pour réclamer l'application de l'obligation de paix sociale en vertu de l'art. 21 de la loi n $18566^{1}$ ou des dispositions conventionnelles.

4) La quatrième et dernière tendance à la judiciarisation est liée aux actions de amparo ${ }^{2}$ engagées suite à l'occupation de l'établissement comme moyen d'exercer le droit de grève. Dans ce type d'action judiciaire, il faut distinguer plusieurs cas. Parfois, c'est l'employeur ou le propriétaire de l'entreprise qui réclame l'expulsion des occupants au titre de son droit de propriété. Selon les tribunaux civils - auprès desquels ces revendications sont formulées du fait de leur "compétence résiduelle $»^{3}$ - le droit de propriété n'est pas affecté lorsque des syndicalistes occupent l'entreprise car ces derniers ne le font pas dans l'idée de la « diriger » puisqu'ils n'en ont pas l'autorisation (article 4.d du décret n 165/2006).

Récemment, l'annonce par le syndicat de sa décision d'occuper une entreprise et de la gérer a suffi à justifier une décision inédite en faveur d'une entreprise (Montevideo Gas SA) interdisant au syndicat (Unión Autónoma de Obreros y Empleados del Gas) d'« empêcher l'entrée et la sortie des autres travailleurs et membres de l'entreprise vers le lieu d'exercice de leurs activités, et de prendre en charge l'activité commerciale de l'entreprise, y compris de contacter les clients et fournisseurs en menaçant de mettre en place le contrôle ouvrier ».

Dans la plupart des cas, ce sont les travailleurs non-grévistes qui engagent des actions de amparo afin de faire valoir leur liberté de travail ou leur liberté de ne pas adhérer à un syndicat. Il s'agit véritablement du cas le plus complexe, puisque le conflit n'oppose pas les «droits du travail » (des grévistes) aux « droits patrimoniaux » (droits de propriété), comme c'est le cas lorsque le recours de amparo est à l'initiative de l'employeur, mais oppose plutôt deux aspects du droit du travail, à savoir la liberté de travail contre le droit de grève via l'occupation du lieu de travail.

1 Selon la loi, " pendant la durée de validité des conventions collectives, les parties s'engagent à ne pas promouvoir d'actions contraires à ce qui a été convenu et à n'appliquer aucune mesure coercitive en ce sens. Cette clause s'applique à toutes les questions qui ont fait l'objet de négociation et qui ont été traitées dans la convention ». Elle ne tient pas compte de la participation aux actions nationales organisées par les syndicats. Afin de résoudre les controverses relatives à l'interprétation de la convention, cette dernière doit instaurer des procédures visant à éliminer toutes les possibilités de négociation directe entre les parties, puis avec l'intervention de l'autorité ministérielle compétente, à éviter les conflits, les actions et les effets générés. Le non-respect des dispositions du premier alinéa du présent article, en l'absence d'une procédure établie par les parties, peut entraîner la résiliation de la convention, qui doit être prononcée par la juridiction du travail.

2 Le recours de amparo en Uruguay, prévu par la loi nº 16011 du 19 décembre 1988, vise à garantir à «toute personne physique ou morale, publique ou privée, la possibilité d'introduire un recours de amparo contre tout acte, omission ou fait des autorités publiques, semi publiques ou de particuliers, de nature, dans l'immédiat ou un futur proche, à léser, restreindre, altérer ou menacer, de façon manifestement illégitime, des droits ou libertés expressément ou implicitement consacrés par la Constitution ». Le recours de amparo se justifie dès lors qu'il n'existe pas d'autre moyen judiciaire ou administratif - ou si ceux-ci sont inefficaces - pour faire respecter ces droits. La procédure consiste en une audience durant trois jours, qui peuvent être prolongés de trois jours très exceptionnellement.

3 Pour rappel, les tribunaux du travail ne sont compétents qu'en matière de conflits individuels du travail (art. 2 de la loi $n^{\circ} 18572$ ). 
Cette jurisprudence des tribunaux civils a connu une évolution tendant à restreindre l'occupation du lieu de travail. Alors qu'elle se limitait initialement à renvoyer les occupants, elle est plus récemment allée jusqu'à interdire l'occupation ou les piquets de grève en invoquant notamment des mesures de précaution. Si cette question mériterait de faire l'objet d'une enquête empirique afin de mettre en lumière certaines pratiques professionnelles et leur influence sur les relations de travail, il ne faut pas oublier que, dans la plupart des cas, ce sont les cabinets d'avocats des entreprises elles-mêmes qui fournissent une assistance juridique aux non-grévistes, ce qui crée des doutes sur l'absence de pression sur les nongrévistes de la part de l'employeur pour mettre fin à l'occupation des lieux.

Pour conclure, la judiciarisation des conflits collectifs du travail se traduit actuellement de plusieurs manières dans le domaine des relations de travail en Uruguay si bien que le problème ne se limite pas seulement à l'expulsion des grévistes qui occupent le lieu de travail, mais porte aussi sur des questions comme le choix des organisations les plus représentatives pour la négociation collective dans les entreprises publiques, la nature des droits des organisations minoritaires, la protection du droit à l'action syndicale, etc. La généralisation du recours de amparo pour faire valoir le droit à la liberté de travail en cas d'occupation du lieu de travail laisse à penser que nous avons affaire à une jurisprudence hégémonique faisant prévaloir le droit individuel au travail sur le droit à la grève. II est difficile de prévoir la manière dont ces formes de judiciarisation vont évoluer. Ce qui est certain, c'est qu'elles ont progressé en raison de la concomitance de trois facteurs: les politiques visant à promouvoir la négociation collective tripartite, la complexité croissante des façons d'exercer le droit de grève, et la croissance de la syndicalisation ces dernières années. La judiciarisation des conflits collectifs semble être une conséquence inéluctable de l'interaction de ces trois facteurs. 